\title{
Symmetry and magnitude of spin-orbit torques in ferromagnetic heterostructures
}

\author{
Kevin Garello*, loan Mihai Miron², Can Onur Avci', Frank Freimuth³, Yuriy Mokrousov³, \\ Stefan Blügel ${ }^{3}$, Stéphane Auffret ${ }^{2}$, Olivier Boulle ${ }^{2}$, Gilles Gaudin² and Pietro Gambardella ${ }^{1,4,5 \star}$
}

\begin{abstract}
Recent demonstrations of magnetization switching induced by in-plane current injection in heavy metal/ferromagnetic heterostructures have drawn increasing attention to spin torques based on orbital-to-spin momentum transfer. The symmetry, magnitude and origin of spin-orbit torques (SOTs), however, remain a matter of debate. Here we report on the three-dimensional vector measurement of SOTs in $\mathrm{AlO}_{x} / \mathrm{Co} / \mathrm{Pt}$ and $\mathrm{MgO} / \mathrm{CoFeB} / \mathrm{Ta}$ trilayers using harmonic analysis of the anomalous and planar Hall effects. We provide a general scheme to measure the amplitude and direction of SOTs as a function of the magnetization direction. Based on space and time inversion symmetry arguments, we demonstrate that heavy metal/ferromagnetic layers allow for two different SOTs having odd and even behaviour with respect to magnetization reversal. Such torques include strongly anisotropic field-like and spin transfer-like components, which depend on the type of heavy metal layer and annealing treatment. These results call for SOT models that go beyond the spin Hall and Rashba effects investigated thus far.
\end{abstract}

M emory and logic spintronic devices rely on the generation of spin torques to control the magnetization of nanoscale elements using electric currents ${ }^{1,2}$. Conventionally, such torques have been associated with the transfer of spin angular momentum between a 'polarizer' and a 'free' ferromagnetic layer separated by a non-magnetic spacer, mediated by a spin-polarized current flowing perpendicular to the two layers ${ }^{2,3}$. Recently, however, experiments ${ }^{4-13}$ and theory ${ }^{14-27}$ have pointed out alternative mechanisms to produce spin torques that do not require a polarizer ferromagnetic layer. These mechanisms, which include the spin $\mathrm{Hall}^{28}$, Rashba ${ }^{29}$ and Dresselhaus ${ }^{30}$ effects, exploit the coupling between electron spin and orbital motion to induce non-equilibrium spin accumulation, which eventually gives rise to a torque on the magnetization via the spin transfer between $s$ and $d$ electrons $^{31,32}$. Henceforth, we refer to such phenomena as spin-orbit torques (SOTs) to underline their common link to the spin-orbit interaction.

Of particular relevance for magnetization switching, experiments on $\mathrm{AlO}_{x} / \mathrm{Co} / \mathrm{Pt}$ heterostructures have shown that current injection in the plane of the layers induces a spin accumulation component transverse to the current, $\delta \mathbf{m}^{\perp} \approx \mathbf{z} \times \mathbf{j}$ (refs 5,6), as well as a longitudinal one that rotates with the magnetization in the plane defined by the current and the $z$-axis of the stack, $\delta \mathbf{m}^{\|} \approx(\mathbf{z} \times \mathbf{j}) \times \mathbf{m}$ (refs 9,33), where $\mathbf{j}$ and $\mathbf{m}$ are unit vectors that denote the current density and magnetization direction, respectively. Because of the exchange interaction between $s$ and $d$ electrons, these components produce two effective magnetic fields, $\mathbf{B}^{\perp} \approx \delta \mathbf{m}^{\perp}$ and $\mathbf{B}^{\|} \approx \delta \mathbf{m}^{\|}$, or, equivalently, a field-like torque $\mathbf{T}^{\perp} \approx \mathbf{m} \times \delta \mathbf{m}^{\perp}$ and a spin transfer-like torque $\mathbf{T}^{\|} \approx \mathbf{m} \times \delta \mathbf{m}^{\|}$. If $\mathbf{j}$ is injected along $\mathbf{x}$, these torques correspond to $\mathbf{T}^{\perp} \approx \mathbf{m} \times \mathbf{y}$ and $\mathbf{T}^{\|} \approx \mathbf{m} \times(\mathbf{y} \times \mathbf{m})$, respectively. Several studies have shown that $\mathbf{T}^{\|}$is strong enough to reverse the magnetization of high-coercivity ferromagnetic layers with both perpendicular ${ }^{9,33,34}$ and in-plane $e^{35}$ anisotropy for current densities of the order of $10^{7}-10^{8} \mathrm{~A} \mathrm{~cm}^{-2}$, raising interest in SOTs for technological applications. For example, it has been proposed $9,36,37$ and demonstrated ${ }^{35}$ that $\mathbf{T}^{\|}$can be used to induce switching of magnetic tunnel junction devices using a three-terminal configuration, where the read and write current paths are separated to avoid damage to the tunnel barrier.

On the theoretical side, two apparently contrasting pictures have emerged: one based on the bulk spin Hall effect (SHE) in the heavy metal layer as the sole source of spin accumulation, ${ }^{9,20,23-25,33,35}$ and the other on Rashba-type effective fields and spin-dependent scattering, which take place at the interface between the heavy metal and the ferromagnetic layer, ${ }^{91-26}$. Both pictures lead to qualitatively equivalent expressions for $\mathrm{T}^{\perp}$ and $\mathrm{T}^{\|}$(refs 23-25) but differ in the relative magnitude of the torques, because a pure SHE implies $\mathbf{T}^{\|} \gg \mathbf{T}^{\perp}$, whereas the opposite is expected if only interfacial Rashba fields are considered ${ }^{25}$. Experiments by Liu et al. have shown that the SHE dominates the contribution to $\mathrm{T}^{\|}$and that its sign is reversed in $\mathrm{MgO} / \mathrm{CoFeB} / \mathrm{Ta}$ and $\mathrm{AlO}_{x} / \mathrm{Co} / \mathrm{Pt}$, consistently with the opposite sign of the SHE in $\mathrm{Ta}$ and $\mathrm{Pt}^{33,35}$. Recent data, however, show that the magnitude and even the sign of both $\mathbf{T}^{\|}$ and $\mathrm{T}^{\perp}$ in $\mathrm{MgO} / \mathrm{CoFeB} / \mathrm{Ta}$ depend on the thickness of the $\mathrm{Ta}$ layer ${ }^{38}$, suggesting that different effects contribute to these torques. This state of affairs, together with the lack of consistent methods to measure the torques, makes it hard to optimize the SOT efficacy for applications and reach a consensus on their physical origin.

The purpose of this Article is threefold. First, starting from symmetry arguments, we derive general expressions of the spin accumulation and current-induced SOTs in magnetic heterostructures that are independent of specific physical models. Second, we present a self-consistent, sensitive method to perform three-dimensional vector measurements of SOTs using an a.c. susceptibility technique based on the combination of the 1st and 2nd harmonic contributions of the anomalous Hall (AHE) and planar Hall (PHE) effects. Third, we demonstrate unambiguously the existence of two distinct SOTs that have odd and even symmetry with respect

'Catalan Institute of Nanoscience and Nanotechnology (ICN2), E-08193 Barcelona, Spain, ${ }^{2}$ SPINTEC, UMR-8191, CEA/CNRS/UJF/GINP, INAC, F-38054 Grenoble, France, ${ }^{3}$ Peter Grünberg Institut and Institute for Advanced Simulation, Forschungszentrum Jülich and JARA, 52425 Jülich, Germany, ${ }^{4}$ Institució Catalana de Recerca i Estudis Avançats (ICREA), E-08010 Barcelona, Spain, ${ }^{5}$ Department of Materials, ETH Zurich, CH-8093 Zurich, Switzerland.

`e-mail: kevin.garello@mat.ethz.ch; pietro.gambardella@mat.ethz.ch 
a

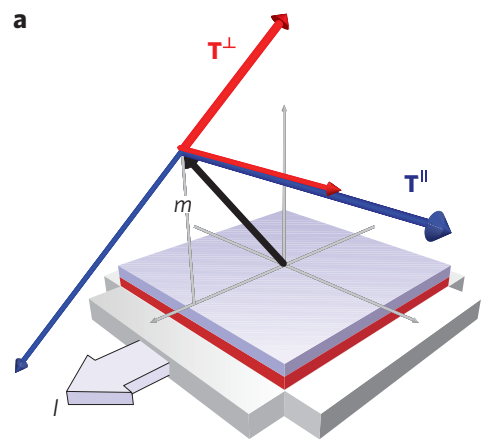

d

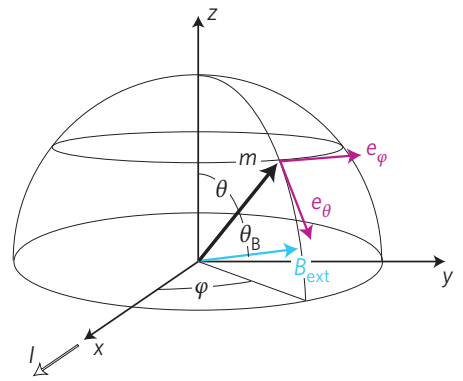

b

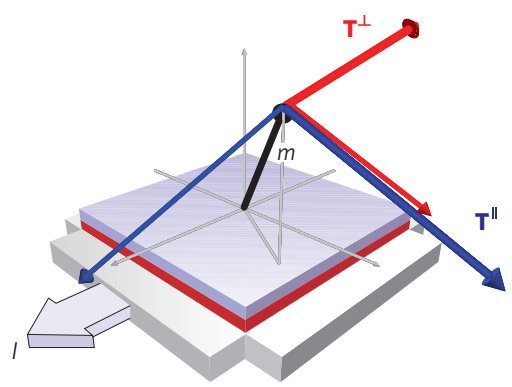

e

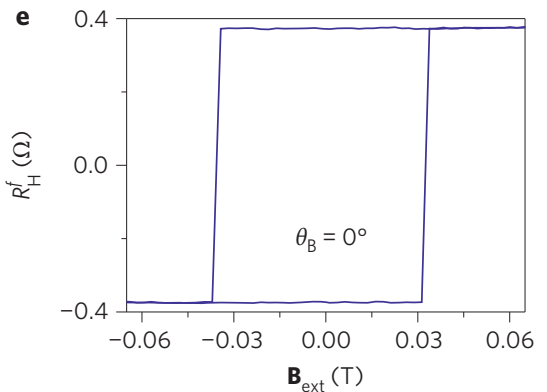

c
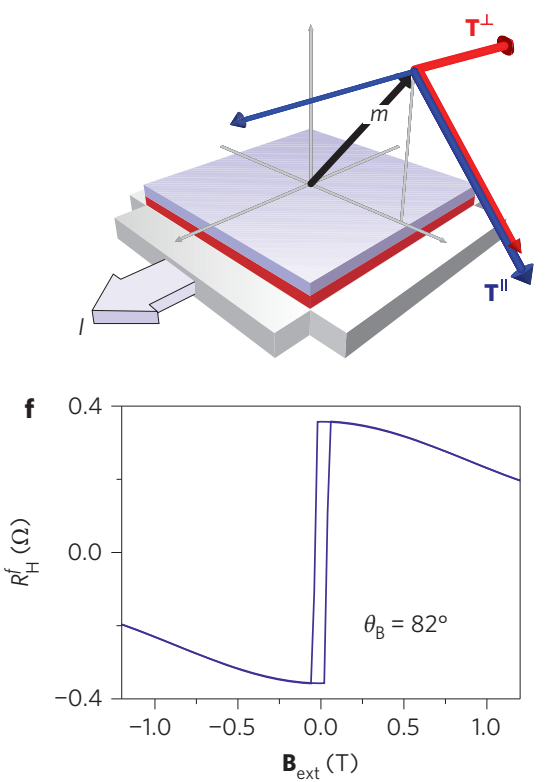

Figure 1 | Torque schematics and magnetization measurements. a-c, $\mathrm{AlO}_{x} / \mathrm{Co} / \mathrm{Pt}$ Hall cross with current and voltage leads. The thick arrows indicate the direction and amplitude of $\mathbf{T}^{\perp}$ (red) and $\mathbf{T}^{\|}$(blue) for $\varphi=0^{\circ}$ (a), $\varphi=60^{\circ}$ (b) and $\varphi=90^{\circ}$ (c). The thin arrows indicate the equivalent fields $\mathbf{B}^{\perp}$ (red) and $\mathbf{B}^{\|}$ (blue). $\mathbf{d}$, Coordinate system. e,f, $m_{z}$ measured by the first harmonic Hall resistance $R_{\mathrm{H}}^{f}$ as a function of $B_{\text {ext }}$ applied parallel to the easy axis $\left(\theta_{\mathrm{B}}=0^{\circ}\right)(\mathbf{e})$ and close to in-plane $\left(\theta_{\mathrm{B}}=82^{\circ}, \varphi=0^{\circ}\right)(\mathbf{f})$.

to the inversion of the magnetization and include, but are not limited to, $\mathbf{T}^{\perp} \approx \mathbf{m} \times \mathbf{y}$ and $\mathbf{T}^{\|} \approx \mathbf{m} \times(\mathbf{y} \times \mathbf{m})$ (Fig. 1a-c). We find strongly anisotropic SOT components that have not been observed to date, which depend on the $x$ and $y$ projections of the magnetization in the plane of the current. $\mathbf{T}^{\perp}$ and $\mathbf{T}^{\|}$have comparable magnitude in $\mathrm{AlO}_{x} / \mathrm{Co} / \mathrm{Pt}$ and decrease significantly due to interface diffusion upon annealing. Both $\mathbf{T}^{\perp}$ and $\mathbf{T}^{\|}$reverse sign and are dominated by anisotropy effects in $\mathrm{MgO} / \mathrm{CoFeB} / \mathrm{Ta}$. The picture that emerges from this study is that interfacial effects play a prominent role in determining the magnitude and anisotropy of the torques.

\section{Spin-orbit torque symmetry and effective fields}

SOTs require inversion asymmetry in order to induce net effects on the magnetization, which is usually realized by sandwiching a ferromagnetic layer between two dissimilar layers (Fig. 1). This holds also for torques produced by the SHE, which average to zero in symmetric heterostructures. In Supplementary Section S1 we derive the general expressions for $\delta \mathbf{m}^{\text {土, }}$ and SOTs consistent with the minimal requirements imposed by structure inversion asymmetry, namely rotational invariance around the $z$-axis and mirror symmetry with respect to planes parallel to $z$. We find that the spin accumulation contains magnetization-dependent terms that add to the $\delta \mathbf{m}^{\perp} \approx \mathbf{y}$ and $\delta \mathbf{m}^{\|} \approx \mathbf{y} \times \mathbf{m}$ components considered thus far, which change the symmetry and amplitude of $\mathbf{T}^{\perp}$ and $\mathbf{T}^{\|}$. The quantitative significance of these terms, however, must be established by experiment. Our measurements determine a minimal set of terms required to model the action of the field-like and spin transfer-like torques, namely

$$
\begin{aligned}
\mathbf{T}^{\perp}= & (\mathbf{y} \times \mathbf{m})\left[T_{0}^{\perp}+T_{2}^{\perp}(\mathbf{z} \times \mathbf{m})^{2}+T_{4}^{\perp}(\mathbf{z} \times \mathbf{m})^{4}\right] \\
+ & \mathbf{m} \times(\mathbf{z} \times \mathbf{m})(\mathbf{m} \cdot \mathbf{x})\left[T_{2}^{\perp}+T_{4}^{\perp}(\mathbf{z} \times \mathbf{m})^{2}\right] \\
\mathbf{T}^{\|}= & \mathbf{m} \times(\mathbf{y} \times \mathbf{m}) T_{0}^{\|} \\
& \quad+(\mathbf{z} \times \mathbf{m})(\mathbf{m} \cdot \mathbf{x})\left[T_{2}^{\|}+T_{4}^{\|}(\mathbf{z} \times \mathbf{m})^{2}\right]
\end{aligned}
$$

These torques are, respectively, odd and even with respect to the inversion of $\mathbf{m}$. For the special case $T_{n}^{\perp}=T_{n}^{\|}=0$ for all $n \neq 0$, equations (1) and (2) simplify to $\mathbf{T}^{\perp}=T_{0}^{\perp}(\mathbf{y} \times \mathbf{m})$ and $\mathbf{T}^{\|}=T_{0}^{\|} \mathbf{m} \times(\mathbf{y} \times \mathbf{m})$, which have been obtained theoretically for several models discussed before ${ }^{21-26}$.

For the purpose of comparison with the experiment, we consider here the effective magnetic fields $\mathbf{B}^{\perp}$ and $\mathbf{B}^{\|}$perpendicular to the magnetization that correspond to $\mathbf{T}^{\perp}$ and $\mathbf{T}^{\|}$obtained above. We adopt a spherical coordinate system (Fig. $1 \mathrm{~d})$, where $\mathbf{m}=(\sin \theta$ $\cos \varphi, \sin \theta \sin \varphi, \cos \theta)$ and obtain

$$
\mathbf{B}^{\perp}=-\cos \theta \sin \varphi\left(T_{0}^{\perp}+T_{2}^{\perp} \sin ^{2} \theta+T_{4}^{\perp} \sin ^{4} \theta\right) \mathbf{e}_{\theta}-\cos \varphi T_{0}^{\perp} \mathbf{e}_{\varphi}
$$

$$
\mathbf{B}^{\|}=\cos \varphi\left(T_{0}^{\|}+T_{2}^{\|} \sin ^{2} \theta+T_{4}^{\|} \sin ^{4} \theta\right) \mathbf{e}_{\theta}-\cos \theta \sin \varphi T_{0}^{\|} \mathbf{e}_{\varphi}
$$

Using equations (3) and (4), the action of the current-induced fields on the magnetization can be directly compared to that of a reference external field $\left(\mathbf{B}_{\text {ext }}\right)$ of known magnitude and direction by means of low-frequency susceptibility measurements ${ }^{6,39}$.

\section{Hall measurements of current-induced effective fields}

We studied $\mathrm{AlO}_{x}(2 \mathrm{~nm}) / \mathrm{Co}(0.6 \mathrm{~nm}) / \mathrm{Pt}(3 \mathrm{~nm})$ as a model system, patterned into $1 \times 1$ and $1 \times 0.5 \mu \mathrm{m}^{2}$ rectangular dots (Fig. 1). We used an a.c. current of frequency $f$ to modulate the SOT amplitude and induce small oscillations of $\mathbf{m}$ about its equilibrium direction, defined by $\mathbf{B}_{\text {ext }}$ and the magnetic anisotropy of the trilayer. Such oscillations generate a second-harmonic contribution to the Hall voltage $\left(V_{\mathrm{H}}\right)$, which provides a sensitive way to measure current-induced fields (Supplementary Section S2). In general, $V_{\mathrm{H}}$ depends on $m_{z}$ through the AHE and on the product $m_{x} m_{y}$ through the PHE:

$$
V_{\mathrm{H}}=R_{\mathrm{AHE}} I \cos \theta+R_{\mathrm{PHE}} I \sin ^{2} \theta \sin 2 \varphi
$$



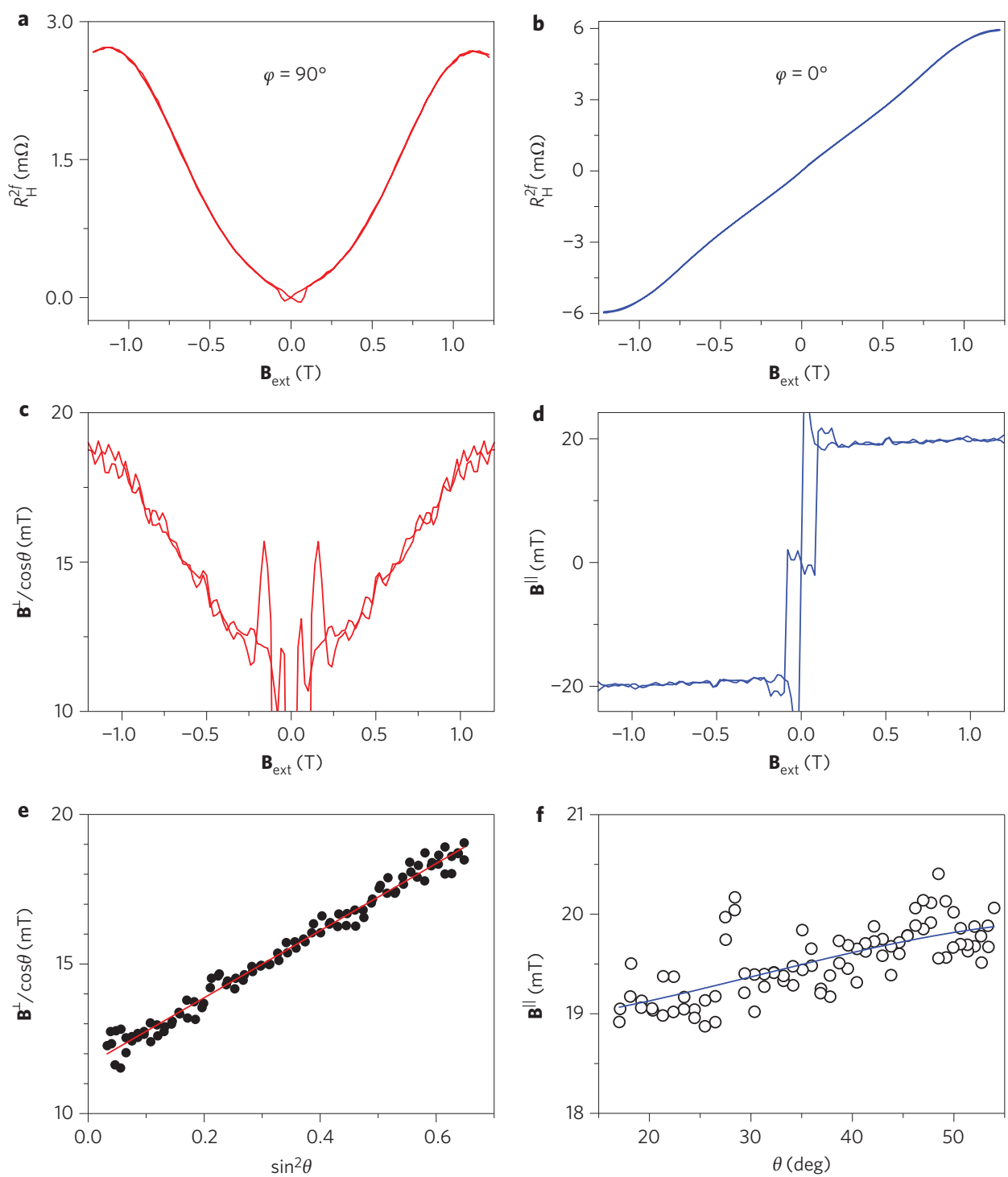

Figure 2 | Second-harmonic Hall resistance and current-induced spin-orbit fields. a,b, $R_{\mathrm{H}}^{2 f}$ measured as a function of $B_{\text {ext }}$ applied at $\theta_{\mathrm{B}}=82^{\circ}$ and $\varphi=90^{\circ}$ (a), and $\theta_{\mathrm{B}}=82^{\circ}$ and $\varphi=0^{\circ} \mathbf{( b )}$. The amplitude of the a.c. current is $1.136 \mathrm{~mA}$. c. Effective field $\mathbf{B}^{\perp} / \cos \theta$ measured at $\varphi=90^{\circ}$ as a function of $B_{\text {ext }} \mathbf{d}$, Effective field $\mathbf{B}^{\|}$measured at $\varphi=0^{\circ}$ as a function of $B_{\text {ext }} \cdot \mathbf{e}, \mathbf{B}^{\perp} / \cos \theta$ measured at $\varphi=90^{\circ}$ as a function of $\sin ^{2} \theta$. The solid line is a fit to $T_{0}^{\perp}+T_{2}^{\perp} \sin ^{2} \theta$ according to equation (3). $\mathbf{f}, \mathbf{B}^{\|}$measured at $\varphi=0^{\circ}$ as a function of $\theta$. The solid line is a fit to $T_{0}^{\|}+T_{2}^{\|} \sin ^{2} \theta+T_{4}^{\|} \sin ^{4} \theta$ according to equation (4). Note that $\left|T^{\perp}\right|=\left|\mathbf{B}^{\perp} / \cos \theta\right|$ for $\varphi=90^{\circ}$ and $\left|T^{\|}\right|=\left|\mathbf{B}^{\|}\right|$for $\varphi=0^{\circ}$.

where $R_{\mathrm{AHE}}$ and $R_{\mathrm{PHE}}$ are the AHE and PHE resistances, respectively, and $I$ is the injected current. In terms of the total Hall resistance $R_{\mathrm{H}}=V_{\mathrm{H}} / I$, the first harmonic term $R_{\mathrm{H}}^{f}=R_{\mathrm{AHE}}^{f}+R_{\mathrm{PHE}}^{f}$ relates to the equilibrium direction of the magnetization and is independent of modulated fields. The second harmonic term $R_{\mathrm{H}}^{2 f}$ measures the susceptibility of the magnetization to the current-induced fields and is given by

$$
\begin{aligned}
R_{\mathrm{H}}^{2 f}= & \left(R_{\mathrm{AHE}}-2 R_{\mathrm{PHE}} \cos \theta \sin 2 \varphi\right) \frac{\mathrm{d} \cos \theta}{\mathrm{d} B_{\mathrm{ext}}} \frac{B_{\theta}}{\sin \left(\theta_{\mathrm{B}}-\theta\right)} \\
& +2 R_{\mathrm{PHE}} \sin ^{2} \theta \cos 2 \varphi \frac{B_{\varphi}}{B_{\mathrm{ext}} \sin \theta_{\mathrm{B}}}
\end{aligned}
$$

where $B_{\theta}$ and $B_{\varphi}$ represent the polar and azimuthal components of the total effective field $\mathbf{B}^{\perp}+\mathbf{B}^{\|}$induced by the current, $\theta_{\mathrm{B}}$ is the polar angle of $\mathbf{B}_{\text {ext }}$, and $\varphi \equiv \varphi_{\mathrm{B}}$ (Fig. 1d). Equation (6) allows us to measure $B_{\theta}$ and $B_{\varphi}$ as a function of $\theta$ and $\varphi$. If $R_{\mathrm{PHE}}=0$, it is straightforward to evaluate $B_{\theta}$ by noting that

$$
R_{\mathrm{AHE}} \frac{\mathrm{d} \cos \theta}{\mathrm{d} B_{\text {ext }}}=\frac{\mathrm{d} R_{\mathrm{H}}^{f}}{\mathrm{~d} B_{\text {ext }}}
$$

Otherwise, $B_{\theta}$ and $B_{\varphi}$ must be evaluated by measuring $V_{\mathrm{H}}$ at $\varphi=0^{\circ}$ and $90^{\circ}$ and fitting $R_{\mathrm{H}}^{2 f}$ using a recursive procedure that accounts for both the $\mathrm{AHE}$ and the $\operatorname{PHE}\left(R_{\mathrm{AHE}}=0.72 \Omega\right.$ and $R_{\mathrm{PHE}}=0.09 \Omega$ for the sample presented in Figs $1-4$ ). This method has been validated by numerical macrospin simulations as well as by applying external a.c. fields in phase and antiphase with the current, the amplitude of which was recovered using equation (6) (Supplementary Sections S4 and S6).

Figure 1e,f shows $R_{\mathrm{H}}^{f}$ as a function of $\mathbf{B}_{\text {ext }}$ applied out of plane $\left(\theta_{\mathrm{B}}=0^{\circ}\right)$ and nearly in-plane $\left(\theta_{\mathrm{B}}=82^{\circ}\right)$, respectively. The curves, proportional to $m_{z}$, are characteristic of $\mathrm{AlO}_{x} / \mathrm{Co} / \mathrm{Pt}$ layers with strong perpendicular magnetic anisotropy. The slow and reversible 

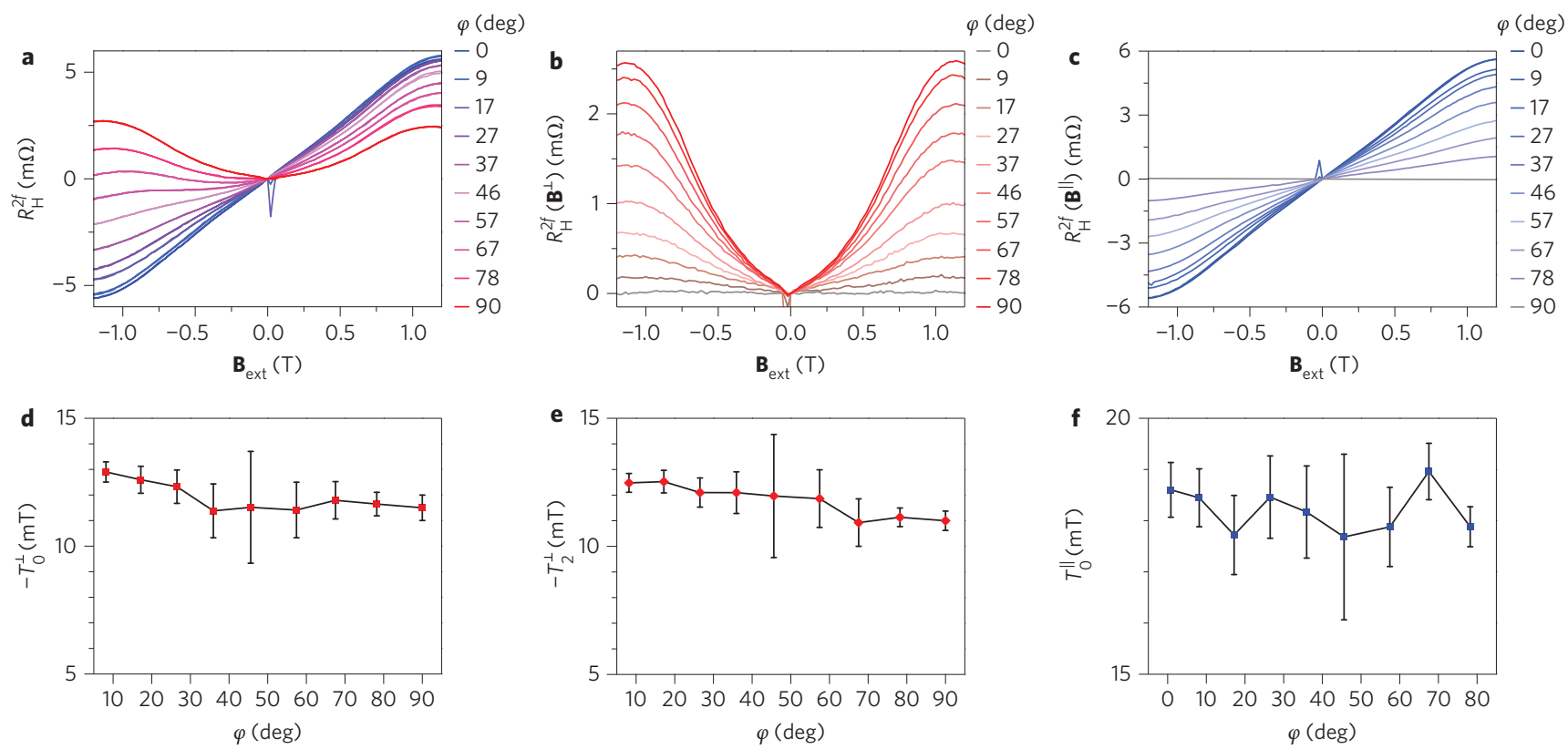

Figure 3 | Angular dependence of the Hall resistance and SOT components. a, $R_{\mathrm{H}}^{2 f}$ as a function of $B_{\text {ext }}$ applied at $\theta_{\mathrm{B}}=82^{\circ}$ measured for different in-plane orientations of the magnetization. $\mathbf{b}, \mathbf{c}$, Symmetric $R_{H}^{2 f}\left(\mathbf{B}^{\perp}\right)(\mathbf{b})$ and antisymmetric $R_{H}^{2 f}\left(\mathbf{B}^{\|}\right)(\mathbf{c})$ components of $R_{\mathrm{H}}^{2 f}$. $\mathbf{d}-\mathbf{f}$, SOT components $T_{0}^{\perp}(\mathbf{d}), T_{2}^{\perp}(\mathbf{e})$ and $T_{0}^{\|}$ (f) as a function of $\varphi$. The error bars represent the experimental errors, which are mostly due to the uncertainty of the PHE measurements. The amplitude of the ac current is $1.136 \mathrm{~mA}$.
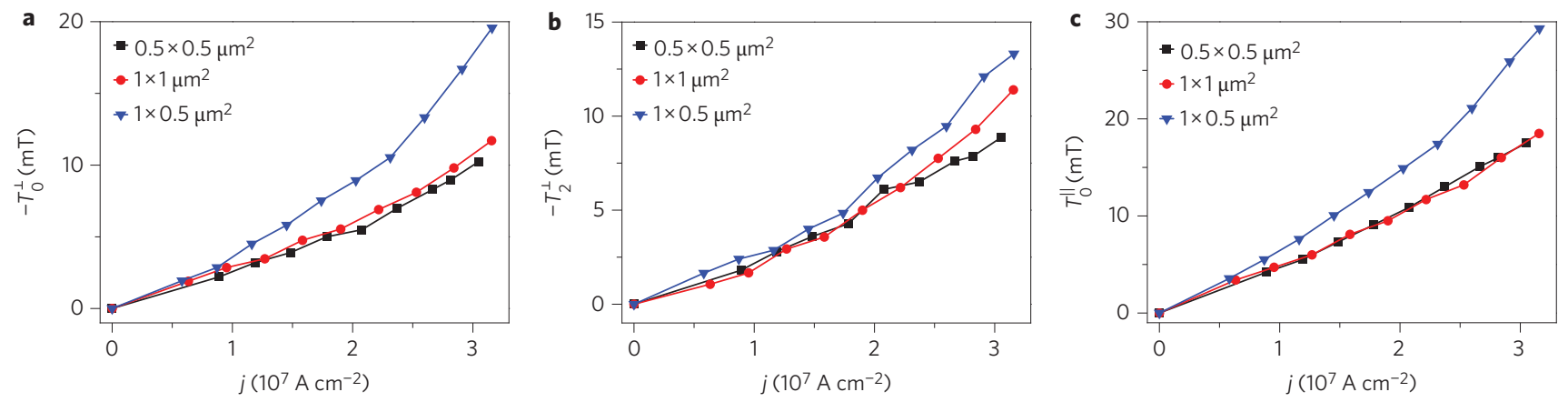

Figure 4 | Dependence of the field-like and spin transfer-like SOT components on the injected current density. a-c, $T_{0}^{\perp}$ (a), $T_{2}^{\perp}$ (b) and $T_{0}^{\|}$(c) as a function of $j$ for different samples. Red circles and black squares refer to square Hall crosses with $1 \times 1 \mu \mathrm{m}^{2}$ and $0.5 \times 0.5 \mu \mathrm{m}^{2}$ dimensions, respectively. The blue triangles refer to a narrow Hall cross with a $1-\mu \mathrm{m}$-wide current line and $0.5-\mu \mathrm{m}$-wide voltage probes.

decrease of $R_{\mathrm{H}}^{f}$ with increasing in-plane field observed in Fig. If is due to the coherent rotation of the Co magnetization towards the hard plane direction. Figure 2 shows the second-harmonic measurements of $R_{\mathrm{H}}^{2 f}$ as a function of $\mathbf{B}_{\text {ext }}$ applied at $\theta_{\mathrm{B}}=82^{\circ}$, perpendicular $\left(\varphi=90^{\circ}\right.$, Fig. 2a) and parallel $\left(\varphi=0^{\circ}\right.$, Fig. $\left.2 \mathrm{~b}\right)$ to the current. The data are shown after subtraction of sample-dependent contributions to the Hall voltage that are not included in equation (5), namely a constant offset due to the voltage probe asymmetry as well as the anomalous Nernst-Ettinghausen effect (ANE). The ANE can be separately measured, giving a small correction to $R_{\mathrm{H}}^{2 f}$ of the order of $0.1 \mathrm{~m} \Omega$ (Supplementary Section S7). We note that the choice of $\theta_{\mathrm{B}}$ is not critical as long as $\mathbf{B}_{\text {ext }}$ is slightly tilted off-plane, to prevent the formation of magnetic domains. According to equation (6), $R_{\mathrm{H}}^{2 f}$ is mostly sensitive to the effective field components parallel to $\mathbf{e}_{\theta}$, as these affect $m_{z}$ and hence the AHE. Conversely, the components parallel to $\mathbf{e}_{\varphi}$ are measured through the PHE, which is significantly weaker. Thus, $R_{\mathrm{H}}^{2 f}$ measured at $\varphi=90^{\circ}$ reflects mostly $\mathbf{B}^{\perp}$ contributions, whereas $R_{\mathrm{H}}^{2 f}$ measured at $\varphi=0^{\circ}$ reflects mostly $\mathbf{B}^{\|}$terms. This agrees with the even/odd character of $R_{\mathrm{H}}^{2 f}$ measured at $\varphi=90^{\circ} / 0^{\circ}$ with respect to field inversion (Fig. 2a,b), because $\mathbf{B}^{\perp}$ and $\mathbf{B}^{\|}$are even and odd with respect to $\mathbf{m}$, opposite to the torques from which they are derived.

\section{Field-like and spin transfer-like torque components}

The effective fields $\mathbf{B}^{\perp}$ and $\mathbf{B}^{\|}$derived from $R_{\mathrm{H}}^{2 f}$ for $\mathbf{m} / / \mathbf{y}\left(\varphi=90^{\circ}\right)$ and $\mathbf{m} / / \mathbf{x}\left(\varphi=0^{\circ}\right)$, respectively, are shown in Fig. 2c,d. We find several interesting features that reveal a more complex scenario than previously anticipated. In particular, $\mathbf{B}^{\perp}$ depends strongly on the direction of $\mathbf{m}$, which is determined here by $\mathbf{B}_{\text {ext. }}$. By converting the field dependence into a $\theta$ dependence using the AHE, we find that $\mathrm{B}^{\perp}$ measured at $\varphi=90^{\circ}$ closely follows the function $-\cos \theta\left(T_{0}^{\perp}+T_{2}^{\perp} \sin ^{2} \theta\right)$, with $T_{0}^{\perp}=-11 . \pm 0.7 \mathrm{mT} \quad$ and $T_{2}^{\perp}=-11.2 \pm 0.6 \mathrm{mT}$ (Fig. 2e). This expression agrees with equation (3), but differs remarkably from that expected from either the Rashba field ${ }^{5,14,24-26}$ or the field-like component of the SHE torque ${ }^{23,25}$ reported in the literature, which imply $T_{2}^{\perp}=0$. We note that $T_{0}^{\perp}$ includes the contribution of the Oersted field produced by the current flowing in the Pt layer, which we estimate as $\mu_{0} I / 2 L=-0.7 \mathrm{mT}$ (antiparallel to $\mathrm{y}$ ), where $L$ is the width of the current line and $\mu_{0}$ the vacuum permeability. 

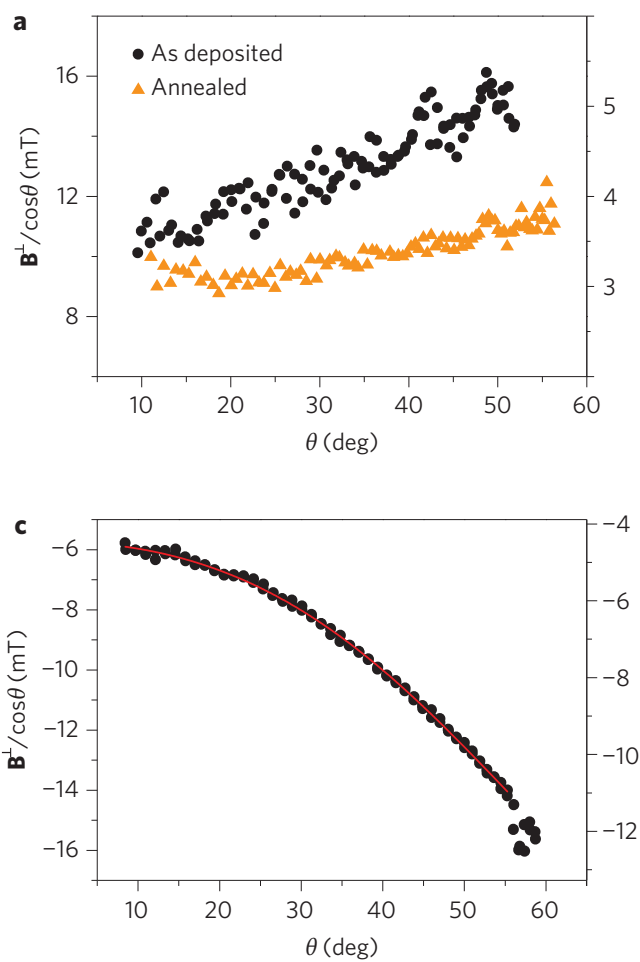

b
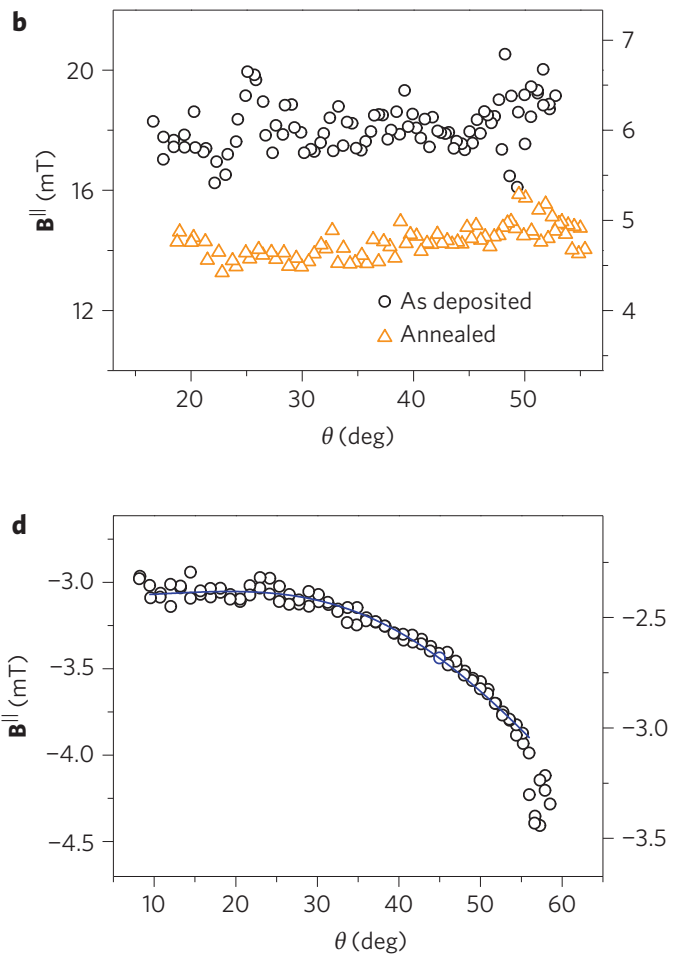

Figure 5 | Effect of thermal annealing and stack composition on current-induced spin-orbit fields. a,b, Effective field $B^{\perp} / \cos \theta$ measured at $\varphi=90^{\circ}$ (a) and effective field $\mathbf{B}^{\|}$measured at $\varphi=0^{\circ}$ (b) in $\mathrm{AlO}_{x} / \mathrm{Co} / \mathrm{Pt}$ as a function of $\theta$. The measurements refer to a $0.5 \times 0.5 \mu \mathrm{m}^{2}$ as-deposited sample (black circles) annealed to $300{ }^{\circ} \mathrm{C}$ (orange triangles). The amplitude of the a.c. current is $540 \mu \mathrm{A}$ and $550 \mu \mathrm{A}$ for the as-deposited and annealed samples, respectively. $\mathbf{c}, \mathbf{d}, \mathbf{B}^{\perp} / \cos \theta$ measured at $\varphi=90^{\circ}$ (c) and $\mathbf{B}^{\|}$measured at $\varphi=0^{\circ}$ (d) for an $\mathrm{MgO} / \mathrm{CoFeB} / \mathrm{Ta}$ Hall bar annealed to $300{ }^{\circ} \mathrm{C}$. The width of the Hall bar is $1 \mu \mathrm{m}$ and the amplitude of the a.c. current is $500 \mu \mathrm{A}$. The scale on the right-hand side of the plots is in $\mathrm{mT}^{\mathrm{T}}$ per $10^{7} \mathrm{~A} \mathrm{~cm}{ }^{-2}$.

The dependence of $\mathbf{B}^{\|}$on the magnetization is remarkably different from $\mathbf{B}^{\perp}$. Figure $2 \mathrm{f}$ shows that $\mathbf{B}^{\|}$measured at $\varphi=0^{\circ}$ is weakly dependent on $\theta$, and is well-approximated by $T_{0}^{\|}+T_{2}^{\|} \sin ^{2} \theta+T_{4}^{\|} \sin ^{4} \theta$, in agreement with equation (4), with $T_{0}^{\|}=19.0 \pm 0.5 \mathrm{mT}, \quad T_{2}^{\|}=2 \pm 1 \mathrm{mT}$ and $T_{4}^{\|}=-1 \pm 1 \mathrm{mT}$. As the higher-order coefficients are small and tend to compensate, B $^{\|}$can be reasonably approximated by a constant value $T_{0}^{\|}$, consistently with previous findings ${ }^{33,34}$. This behaviour is typical of as-deposited $\mathrm{AlO}_{x} / \mathrm{Co} / \mathrm{Pt}$ samples, apart from small changes of the coefficients that we attribute to pattern or material inhomogeneities.

To complete the description of $\mathbf{B}^{\perp}$ and $\mathbf{B}^{\|}$, we performed a series of measurements for different in-plane orientations of $\mathbf{m}$. When $\varphi$ deviates from $0^{\circ}$ or $90^{\circ}, R_{\mathrm{H}}^{2 f}$, shown in Fig. 3a, is given by the linear superposition of two terms $R_{\mathrm{H}}^{2 f}\left(\mathbf{B}^{\perp}\right)+R_{\mathrm{H}}^{2 f}\left(\mathbf{B}^{\|}\right)$, which can be easily separated owing to their even/odd symmetry with respect to the inversion of $\mathbf{m}$. Figure $3 \mathrm{~b}, \mathrm{c}$ shows $R_{\mathrm{H}}^{2 f}\left(\mathbf{B}^{\perp}\right)$ and $R_{\mathrm{H}}^{2 f}\left(\mathbf{B}^{\|}\right)$as a function of $\varphi$. The lineshape of $R_{\mathrm{H}}^{2 f}\left(\mathbf{B}^{\perp}\right)$ is similar to $R_{\mathrm{H}}^{2 f}$ measured at $\varphi=90^{\circ}$ (Fig. 2a), whereas $R_{\mathrm{H}}^{2 f}\left(\mathbf{B}^{\|}\right)$is similar to $R_{\mathrm{H}}^{2 f}$ measured at $\varphi=0^{\circ}$ (Fig. 2b). The amplitude of $R_{\mathrm{H}}^{2 f}\left(\mathbf{B}^{\perp}\right)$ increases whereas $R_{\mathrm{H}}^{2 f}\left(\mathbf{B}^{\|}\right)$decreases as $\varphi$ goes from $0^{\circ}$ to $90^{\circ}$. From these curves we obtain that the polar component of $\mathbf{B}^{\perp}$ scales proportionally to $\sin \varphi$, whereas the polar component of $\mathbf{B}^{\|}$scales as $\cos \varphi$, in agreement with equations (3) and (4), respectively. This implies that, within the error of our data, the SOT coefficients $T_{0}^{\perp}, T_{2}^{\perp}$ and $T_{0}^{\|}$are independent of $\varphi$ (Fig. 3d-f), in agreement with the superposition principle for the current and the resulting linear-response torques.

\section{Torque-to-current ratios}

Figure 4 shows that the amplitudes of $\mathbf{T}^{\perp}$ and $\mathbf{T}^{\|}$scale linearly with the current up to $j=1.5 \times 10^{7} \mathrm{~A} \mathrm{~cm}^{-2}$. Above this value, we observe a nonlinear increase of the coefficients $T_{0}^{\perp}, T_{2}^{\perp}$ and $T_{0}^{\|}$, which we attribute to Joule heating. At the maximum current density used in this study $\left(3.15 \times 10^{7} \mathrm{~A} \mathrm{~cm}^{-2}\right)$, heating induces a reduction of the AHE $(-3.5 \%)$ and magnetic anisotropy $(-13 \%)$, as well as an increase in the resistivity of the layers $(+13 \%)$. We caution that these effects can alter the intrinsic SOT/current ratio and also introduce experimental artefacts (Supplementary Section S12).

Our measurements also offer quantitative insight into the magnitude of the different SOT components. We first discuss $\mathbf{T}^{\perp}$ From the initial slope of the data in Fig. 4, we find that the torque/ current ratios corresponding to $T_{0}^{\perp}$ and $T_{2}^{\perp}$ are $-3.2 \pm 0.2$ and $-2.3 \pm 0.2 \mathrm{mT}$ per $10^{7} \mathrm{~A} \mathrm{~cm}^{-2}$, respectively. This corrects our previous estimate of $\mathbf{T}^{\perp}$ based on current-induced domain nucleation ${ }^{5}$, which largely overestimated $T_{0}^{\perp}$ due to heat-assisted magnetization reversal $^{6}$ and neglect of $T_{2}^{\perp}$. Moreover, our measurements are quasi-static and extend well into the low-current regime, proving that $\mathbf{T}^{\perp}$ does not result from the spin Hall torque dynamics at high current. This hypothesis was suggested by Liu et al., who reported no evidence of $\mathrm{T}^{\perp}$ in $\mathrm{AlO}_{x} / \mathrm{Co} / \mathrm{Pt}$ within a sensitivity of $1.3 \mathrm{mT}$ per $10^{7} \mathrm{~A} \mathrm{~cm}^{-2}$ (ref. 33). We suggest that the negative result of Liu et al. might be partly due to the different preparation of the $\mathrm{AlO}_{x} / \mathrm{Co} / \mathrm{Pt}$ stack (oxidized in air and annealed up to $350{ }^{\circ} \mathrm{C}$ ) and, possibly, to the different measurement method (see Supplementary Section S10 for a comparison of a.c. and d.c. measurements).

We next consider $\mathbf{T}^{\|}$, fitting the low-current data $\left(j<1.5 \times 10^{7} \mathrm{~A} \mathrm{~cm}^{-2}\right)$ in Fig. $4 c$. We obtain $T_{0}^{\|}=5.0 \pm 0.2 \mathrm{mT}$ per $10^{7} \mathrm{~A} \mathrm{~cm}^{-2}$ for the square Hall crosses (circles and squares in Fig. 4c). This represents a lower bound for the torque amplitude due to current dispersion in the voltage probes, which can reach up to $23 \%$ of the total current ${ }^{40}$. Measurements of Hall crosses with narrower voltage probes $(0.5 \mu \mathrm{m}$ instead of $1 \mu \mathrm{m})$ give consistently higher torque/current ratios, namely $T_{0}^{\perp}=-4.0 \pm 0.3$, 
$T_{2}^{\perp}=-2.7 \pm 0.1$ and $T_{0}^{\|}=6.9 \pm 0.3 \mathrm{mT}$ per $10^{7} \mathrm{~A} \mathrm{~cm}^{-2}$ (Fig. 4, triangles, and Supplementary Section S8). The latter value of $T_{0}^{\|}$ is about four times larger than that reported by Liu et al. for $\mathrm{AlO}_{x} / \mathrm{Co} / \mathrm{Pt}\left(1.7 \mathrm{mT} \text { per } 10^{7} \mathrm{~A} \mathrm{~cm}^{-2}\right)^{33}$, and also larger compared to $\mathrm{MgO} / \mathrm{CoFeB} / \mathrm{Ta}\left(4 \mathrm{mT} \text { per } 10^{7} \mathrm{~A} \mathrm{~cm}^{-2}\right)^{35}$. Supposing that $\mathrm{T}^{\|}$ originates uniquely from the bulk SHE of Pt, using the model of ref. 33 and $\lambda_{\mathrm{Pt}}=1.4 \mathrm{~nm}$ for the spin diffusion length ${ }^{41}$, our measurement of $T_{0}^{\|}$implies a spin Hall angle $\vartheta_{\mathrm{SH}}=0.16$, which is comparable to $\mathrm{Ta}$ (ref. 35) and twice the largest value reported for Pt (ref. 41). Taking $\lambda_{\mathrm{Pt}}=10 \mathrm{~nm}$, as discussed in a recent report ${ }^{42}$, would push $\vartheta_{\mathrm{SH}}$ to even higher values, suggesting that either $\vartheta_{\mathrm{SH}}$ is larger than expected or additional effects contribute to $\mathbf{T}^{\|}$.

\section{Torque dependence on interface and material parameters} To investigate how the SOTs depend on the quality of the $\mathrm{AlO}_{x} / \mathrm{Co} / \mathrm{Pt}$ interfaces, we measured $\mathbf{B}^{\perp}$ and $\mathbf{B}^{\dagger}$ on trilayers annealed to $300{ }^{\circ} \mathrm{C}$ for $30 \mathrm{~min}$ in vacuum. We find that annealing induces a significant degradation of the SOT amplitude, corresponding to a reduction of $T_{0}^{\perp}, T_{2}^{\perp}$ and $T_{0}^{\|}$by $\sim 17 \%, 60 \%$ and $23 \%$, respectively (Fig. 5a,b). The resistivity, which is $36 \mu \Omega \mathrm{cm}$ in the as-deposited samples, increases by $\sim 7 \%$, whereas the AHE goes from 0.80 to $1.14 \Omega$. This is consistent with previous measurements of annealed $\mathrm{AlO}_{x} / \mathrm{Co} / \mathrm{Pt}$ trilayers ${ }^{43}$, where the AHE increase was attributed to the diffusion of $\mathrm{Pt}$ atoms into the Co layer. Because annealing above $250{ }^{\circ} \mathrm{C}$ is known to induce mixing of Co and $\mathrm{Pt}$ and affect the oxidation of the $\mathrm{AlO}_{x} / \mathrm{Co}$ interface ${ }^{43,44}$, we conclude that both $\mathbf{T}^{\perp}$ and $\mathbf{T}^{\|}$are very sensitive to the interface quality of the trilayers.

The results on $\mathrm{MgO}(2 \mathrm{~nm}) / \mathrm{CoFeB}(0.9 \mathrm{~nm}) / \mathrm{Ta}(3 \mathrm{~nm})$ layers, also annealed to $300{ }^{\circ} \mathrm{C}$ in vacuum, are shown in Fig. 5 c,d. By comparison with Fig. 5a,b, it is evident that $\mathbf{B}^{\perp}$ and $\mathbf{B}^{\|}$reverse sign relative to $\mathrm{AlO}_{x} / \mathrm{Co} / \mathrm{Pt}$, consistently with previous studies ${ }^{35,38}$. However, the strong $\theta$ dependence of both fields, not observed before, reveals that the SOT anisotropy is a general effect that is not unique to $\mathrm{AlO}_{x} / \mathrm{Co} / \mathrm{Pt}$. The fits of $\mathbf{B}^{\perp}$ and $\mathbf{B}^{\|}$according to equations (3) and (4) give $T_{0,2,4}^{\perp}=4.5 \pm 0.1, \quad 5.6 \pm 0.2$ and $5.9 \pm 0.3$ and $T_{0,2,4}^{\|}=-2.4 \pm 0.1,0.4 \pm 0.4$ and $-2.0 \pm 0.4 \mathrm{mT}$ per $10^{7} \mathrm{~A} \mathrm{~cm}^{-2}$. Thus, both second- and fourth-order terms of amplitude comparable to the zeroth order are required to model the torque angular dependence in $\mathrm{MgO} / \mathrm{CoFeB} / \mathrm{Ta}$. We note also that the field-like terms are considerably larger than the spin-transfer like ones, unlike for perpendicular current injection in metallic spin valve systems ${ }^{45}$.

In conclusion, general symmetry arguments show that $\mathbf{T}^{\perp}$ and $\mathbf{T}^{\|}$ can have a complex vector dependence on the direction of the magnetization. This work provides the first evidence for this effect as well as a method to measure $\mathbf{T}^{\perp}$ and $\mathbf{T}^{\|}$, and their dependence on the magnetization in vector form. We find that there are significant deviations from the SOT models considered so far based on the Rashba effect and SHE. In the case of $\mathrm{AlO}_{x} / \mathrm{Co} / \mathrm{Pt}$, the largest deviations are observed for $\mathbf{T}^{\perp}$ due to terms proportional to $T_{2}^{\perp}$ in equation (1). Thus, the effective field $\mathbf{B}^{\perp}$ generated by the current includes magnetization-dependent components perpendicular to the $y$-axis, whereas the Rashba model can only explain components parallel to $y$. This suggests that the previous picture of $\mathbf{T}^{\perp}$ induced by a Rashba field of constant magnitude ${ }^{9,21-26}$ or the field-like component of the spin Hall torque t3,25 $^{23}$ has to be extended by a calculation of the torque based on a realistic description of the electronic structure. In the case of $\mathrm{MgO} / \mathrm{CoFeB} / \mathrm{Ta}$, both $\mathrm{T}^{\perp}$ and $\mathrm{T}^{\|}$present strong anisotropic components, which maximize the torques when the magnetization lies in the plane of the ferromagnetic layer. Tuning of the vector properties of SOTs may play a crucial role in developing spintronic devices where different magnetic states are induced by distinct SOT components.

\section{Methods}

The samples were fabricated from $\mathrm{Al}(1.6 \mathrm{~nm}) / \mathrm{Co}(0.6 \mathrm{~nm}) / \mathrm{Pt}(3 \mathrm{~nm})$ and $\mathrm{MgO}(2 \mathrm{~nm}) / \mathrm{CoFeB}(0.9 \mathrm{~nm}) / \mathrm{Ta}(3 \mathrm{~nm})$ layers deposited on a thermally oxidized silicon wafer by d.c. magnetron sputtering. The deposition rates were $0.05 \mathrm{~nm} \mathrm{~s}^{-1}$ (Co and $\mathrm{Al}$ ), $0.15 \mathrm{~nm} \mathrm{~s}^{-1}$ (Ta) and $0.1 \mathrm{~nm} \mathrm{~s}^{-1}$ (Pt, Mg) at an Ar pressure of $2 \times$ $10^{-3}$ mbar. After deposition, the $\mathrm{Al} / \mathrm{Co} / \mathrm{Pt}$ films were oxidized by exposure to a radiofrequency oxygen plasma at a pressure of $3 \times 10^{-3}$ mbar and a radiofrequency power of $10 \mathrm{~W}$ for $29 \mathrm{~s}$. $\mathrm{Mg} / \mathrm{CoFeB} / \mathrm{Ta}$ was naturally oxidized in an oxygen pressure of $150 \mathrm{mbar}$ for $10 \mathrm{~s}$. The $\mathrm{AlO}_{x} / \mathrm{Co} / \mathrm{Pt}$ films were patterned by electron-beam lithography and ion beam etching into 1,000- and 500-nm-square $\mathrm{AlO}_{x} / \mathrm{Co}$ dots and Pt Hall crosses. The typical resistance of these devices is $3-4 \mathrm{k} \Omega$ and is mostly due to the thin $\mathrm{Pt}$ contact leads, whereas the resistivity of $\mathrm{AlO}_{x} / \mathrm{Co} / \mathrm{Pt}$ is $36 \mu \Omega \mathrm{cm}$. The $\mathrm{MgO} / \mathrm{CoFeB} / \mathrm{Ta}$ layers were patterned into 1,000-nm-wide Hall bars with $500 \mathrm{~nm}$ voltage branches. The resistivity of our $\mathrm{MgO} / \mathrm{CoFeB} / \mathrm{Ta}$ devices is $184 \mu \Omega \mathrm{cm}$. The Hall voltage measurements were performed at room temperature by using an a.c. current with an amplitude of 200 to $1,136 \mu \mathrm{A}$ modulated at $f=10 \mathrm{~Hz}$. $V_{\mathrm{H}}$ was recorded during sweeps of the external magnetic field for $10 \mathrm{~s}$ at each field step, and fast Fourier transformed to extract $R_{\mathrm{H}}^{f}$ and $R_{\mathrm{H}}^{2 f}$. The torques derived from $R_{\mathrm{H}}^{f}$ and $R_{\mathrm{H}}^{2 f}$ are expressed per unit of magnetic moment, thereby using the same units for torques and effective fields. The values of the current density are calculated assuming homogeneous current distribution in the heavy metal/ferromagnetic layers.

Received 26 July 2012; accepted 25 June 2013;

published online 28 July 2013

\section{References}

1. Chappert, C., Fert, A. \& Van Dau, F. N. The emergence of spin electronics in data storage. Nature Mater. 6, 813-823 (2007).

2. Brataas, A., Kent, A. D. \& Ohno, H. Current-induced torques in magnetic materials. Nature Mater. 11, 372-381 (2012).

3. Ralph, D. C. \& Stiles, M. D. Spin transfer torques. J. Magn. Magn. Mater. 320, 1190-1216 (2008).

4. Chernyshov, A. et al. Evidence for reversible control of magnetization in a ferromagnetic material by means of spin-orbit magnetic field. Nature Phys. 5, 656-659 (2009).

5. Miron, I. M. et al. Current-driven spin torque induced by the Rashba effect in a ferromagnetic metal layer. Nature Mater. 9, 230-234 (2010).

6. Pi, U. H. et al. Tilting of the spin orientation induced by Rashba effect in ferromagnetic metal layer. Appl. Phys. Lett. 97, 162507 (2010).

7. Fang, D. et al. Spin-orbit-driven ferromagnetic resonance. Nature Nanotech. 6 , 413-417 (2011).

8. Suzuki, T. et al. Current-induced effective field in perpendicularly magnetized $\mathrm{Ta} / \mathrm{CoFeB} / \mathrm{MgO}$ wire. Appl. Phys. Lett. 98, 142505 (2011).

9. Miron, I. M. et al. Perpendicular switching of a single ferromagnetic layer induced by in-plane current injection. Nature 476, 189-193 (2011).

10. Kajiwara, Y. et al. Transmission of electrical signals by spin-wave interconversion in a magnetic insulator. Nature 464, 262-266 (2010).

11. Kurebayashi, H. et al. Controlled enhancement of spin-current emission by three-magnon splitting. Nature Mater. 10, 660-664 (2011).

12. Liu, L., Moriyama, T., Ralph, D. C. \& Buhrman, R. A. Spin-torque ferromagnetic resonance induced by the spin Hall effect. Phys. Rev. Lett. 106, 036601 (2011).

13. Demidov, V. E. et al. Magnetic nano-oscillator driven by pure spin current. Nature Mater. 11, 1028-1031 (2012).

14. Manchon, A. \& Zhang, S. Theory of nonequilibrium intrinsic spin torque in a single nanomagnet. Phys. Rev. B 78, 212405 (2008).

15. Obata, K. \& Tatara, G. Current-induced domain wall motion in Rashba spinorbit system. Phys. Rev. B 77, 214429 (2008).

16. Manchon, A. \& Zhang, S. Theory of spin torque due to spin-orbit coupling. Phys. Rev. B 79, 094422 (2009).

17. Garate, I. \& MacDonald, A. Influence of a transport current on magnetic anisotropy in gyrotropic ferromagnets. Phys. Rev. B 80, 134403 (2009).

18. Matos-Abiague, A. \& Rodríguez-Suárez, R. Spin-orbit coupling mediated spin torque in a single ferromagnetic layer. Phys. Rev. B 80, 094424 (2009).

19. Haney, P. M. \& Stiles, M. D. Current-induced torques in the presence of spinorbit coupling. Phys. Rev. Lett. 105, 126602 (2010).

20. Vedyayev, A., Strelkov, N., Chshiev, M., Ryzhanova, N. \& Dieny, B. Spin transfer torques induced by spin Hall effect. Preprint at http://lanl.arXiv.org/abs/1108. 2589v1 (2011).

21. Wang, X. \& Manchon, A. Rashba spin torque in an ultrathin ferromagnetic metal layer. Preprint at http://lanl.arXiv.org/abs/1111.5466 (2011).

22. Wang, X. \& Manchon, A. Diffusive spin dynamics in ferromagnetic thin films with a Rashba interaction. Phys. Rev. Lett. 108, 117201 (2012).

23. Manchon, A. Spin Hall effect versus Rashba torque: a diffusive approach. Preprint at http://lanl.arXiv.org/abs/1204.4869 (2012).

24. Pesin, D. A. \& MacDonald, A. H. Quantum kinetic theory of current-induced torques in Rashba ferromagnets. Phys. Rev. B 86, 014416 (2012).

25. Haney, P. M., Lee, H-W., Lee, K-J., Manchon, A. \& Stiles, M. D. Current induced torques and interfacial spin-orbit coupling: semiclassical modeling. Phys. Rev. B 87, 174411 (2013). 
26. Kim, K-W., Seo, S-M., Ryu, J., Lee, K-J. \& Lee, H-W. Magnetization dynamics induced by in-plane currents in ultrathin magnetic nanostructures with Rashba spin-orbit coupling. Phys. Rev. B 85, 180404 (2012).

27. Van der Bijl, E. \& Duine, R. A. Current-induced torques in textured Rashba ferromagnets. Phys. Rev. B 86, 094406 (2012).

28. Dyakonov, M. I. \& Perel, V. I. Possibility of orienting electron spins with current. JETP Lett. 13, 467-469 (1971).

29. Bychkov, Y. A. \& Rashba, E. I. Properties of a $2 d$ electron-gas with lifted spectral degeneracy. JETP Lett. 39, 78-81 (1984).

30. Dresselhaus, G. Spin-orbit coupling effects in zinc blende structures. Phys. Rev. 100, 580-586 (1955).

31. Zhang, S., Levy, P. \& Fert, A. Mechanisms of spin-polarized current-driven magnetization switching. Phys. Rev. Lett. 88, 236601 (2002).

32. Tserkovnyak, Y., Brataas, A. \& Bauer, G. E. W. Theory of current-driven magnetization dynamics in inhomogeneous ferromagnets. J. Magn. Magn. Mater. 320, 1282-1292 (2008).

33. Liu, L., Lee, O. J., Gudmundsen, T. J., Ralph, D. C. \& Buhrman, R. A. Currentinduced switching of perpendicularly magnetized magnetic layers using spin torque from the spin Hall effect. Phys. Rev. Lett. 109, 096602 (2012).

34. Avci, C. O. et al. Magnetization switching of an $\mathrm{MgO} / \mathrm{Co} / \mathrm{Pt}$ layer by in-plane current injection. Appl. Phys. Lett. 100, 212404 (2012).

35. Liu, L. et al. Spin-torque switching with the giant spin Hall effect of tantalum. Science 336, 555-558 (2012).

36. Gaudin, G., Miron, I. M., Gambardella, P. \& Schuhl, A. Magnetic memory element. WO patent 2012/014131 (2012).

37. Gaudin, G., Miron, I. M., Gambardella, P. \& Schuhl, A. Writable magnetic element. WO patent 2012/014132 (2012).

38. Kim, J. et al. Layer thickness dependence of the current-induced effective field vector in Ta|CoFeB|MgO. Nature Mater. 12, 240-245 (2013).

39. Miron, I. et al. Domain wall spin torquemeter. Phys. Rev. Lett. 102, 137202 (2009).

40. Ibrahim, I. S., Schweigert, V. A. \& Peeters, F. M. Diffusive transport in a Hall junction with a microinhomogeneous magnetic field. Phys. Rev. B 57, 15416-15427 (1998).

41. Liu, L., Buhrman, R. A. \& Ralph, D. C. Review and analysis of measurements of the spin Hall effect in platinum. Preprint at http://lanl.arXiv.org/abs/1111.3702v3 (2011).
42. Niimi, Y. et al. Experimental verification of comparability between spin-orbit and spin-diffusion lengths. Phys. Rev. Lett. 110, 016805 (2013).

43. Rodmacq, B., Manchon, A., Ducruet, C., Auffret, S. \& Dieny, B. Influence of thermal annealing on the perpendicular magnetic anisotropy of $\mathrm{Pt} / \mathrm{Co} / \mathrm{AlOx}$ trilayers. Phys. Rev. B 79, 024423 (2009).

44. Wang, Y. et al. Effect of annealing on the magnetic tunnel junction with $\mathrm{Co} / \mathrm{Pt}$ perpendicular anisotropy ferromagnetic multilayers. J. Appl. Phys. 107, 09c711 (2010).

45. Zimmler, M. et al. Current-induced effective magnetic fields in $\mathrm{Co} / \mathrm{Cu} / \mathrm{Co}$ nanopillars. Phys. Rev. B 70, 184438 (2004).

\section{Acknowledgements}

This work was supported by the European Research Council (StG 203239 NOMAD), the European Commission under the Seventh Framework Programme (GA 318144, SPOT), Ministerio de Economía y Competitividad (ERA-Net EUI2008-03884, MAT2010-15659), Agència de Gestió d'Ajuts Universitaris i de Recerca (2009 SGR 695) and the Agence Nationale de la Recherche (ANR-10-BLANC-1011-3 'SPINHALL'). F.F. and Y.M. acknowledge funding under the HGF-YIG programme VH-NG-513. The samples were patterned at the NANOFAB facility of the Institut Néel (CNRS).

\section{Author contributions}

K.G., I.M.M. and P.G. planned the experiment. I.M.M., C.O.A., G.G. and S.A. fabricated the samples. K.G., I.M.M. and C.O.A. performed the measurements. K.G., I.M.M., C.O.A. and P.G. analysed the data. F.F. derived the general expression for the torques. K.G. and P.G. wrote the manuscript. All authors discussed the results and commented on the manuscript.

\section{Additional information}

Supplementary information is available in the online version of the paper. Reprints and permissions information is available online at www.nature.com/reprints. Correspondence and requests for materials should be addressed to K.G. and P.G.

\section{Competing financial interests}

The authors declare no competing financial interests. 